\title{
Early Diagnosis of Interstitial Pregnancy Associated to Assisted Reproductive Techniques Case Report
}

\section{Mercedes del Pilar Alvarez-Goris ${ }^{1 *}$, Miguel Martinez ${ }^{2}$ and Abraham Zavala-Garcia ${ }^{2}$}

${ }^{1} \mathrm{OB} / \mathrm{GYN}$ Resident, Mexican Faculty of Medicine La Salle University, Mexico

${ }^{2}$ Reproductive Biology, Reproduction and Genetics AGN and Associates, Hospital Angeles del Pedregal, Mexico

${ }^{*}$ Corresponding author: Mercedes del Pilar Alvarez-Goris, OB/GYN Resident, Mexican Faculty of Medicine La Salle University, Mexico, Tel: +5255 35089077; E-mail: goris14@hotmail.com

Received date: March 13, 2017; Accepted date: April 18, 2017; Published date: April 24, 2017

Citation: Alvarez-Goris MP, Martinez M, Zavala-Garcia A (2017) Early Diagnosis of Interstitial Pregnancy Associated to Assisted Reproductive Techniques Case Report. Reproductive Immunol Open Acc 2: 31. doi: 10.21767/2476-1974.100031

Copyright: @ 2017 Alvarez-Goris MP, et al. This is an open-access article distributed under the terms of the Creative Commons Attribution License, which permits unrestricted use, distribution, and reproduction in any medium, provided the original author and source are credited.

\section{Abstract}

Background: $2.4 \%$ of ectopic pregnancies develop in the interstitial portion of the fallopian tube and is associated over to a $2.5 \%$ mortality rate, in contrast to other forms of tubal ectopic pregnancy with a lower mortality rate.

Case Report: A patient with a 5 years history of primary infertility and severe endometriosis undergoes an in vitro fertilization (IVF) after pituitary suppression. Embryos were transferred and a month afterward, ultrasonography examination reported an $18 \mathrm{~mm}$ gestational sac, with an embryo in the left adnexal mass. However, an empty uterine cavity is reported in spite of an elevated human chorionic gonadotropin beta fraction. Because of this a diagnostic/ operative laparoscopy is performed. The histopathology result reports a left interstitial ectopic pregnancy. The patient progresses through an uneventful postoperative period, and is checked-out the next day in proper clinical status.

Conclusion: Ectopic pregnancy is a complication of assisted reproduction techniques and interstitial pregnancy is a rare form of tubal pregnancy posing a diagnostic challenge and a life-threatening condition due to the risk of massive bleeding. Hence, clinical suspicion of an interstitial pregnancy requires a quick intervention to avoid a potentially life-threatening situation.

Keywords: Interstitial pregnancy; Ectopic pregnancy; Pregnancy complications; Tubal pregnancy; Maternal death

\section{Background}

The incidence of tubal sterilization failure is estimated to be between 0.13 and 1.3\%; of these cases, $15-33 \%$ present as ectopic pregnancies. Tubal recanalization and tubal-peritoneal fistulas are the main cause of ectopic pregnancy following tubal sterilization [1]. The incidence of ectopic pregnancy is $1-2 \%$ in all pregnancies [2], and interstitial pregnancy is a rare form of tubal ectopic pregnancy. In fact, only $2.4 \%$ of ectopic pregnancies develop in the interstitial portion of the fallopian tube in one of every 2500-5000 births. Moreover, the mortality rate is over $2.5 \%$; seven times higher than other tubal ectopic pregnancies $[3,4]$. The interstitial portion of the fallopian tube crosses the uterine muscle wall in a transverse direction, originating from the tubal ostium and traversing upwards and laterally in relation to the uterine cavity, up to the isthmic portion of the fallopian tube where it exits from the uterine fundus. This is a highly irrigated region due to the presence of the uterine-ovarian artery anastomosis [3-6]. This portion has a diameter of $0.7 \mathrm{~mm}$ to $1.2 \mathrm{~cm}$, and despite its narrowness, it has a greater capacity of distension before rupture in comparison to distal portions of the fallopian tube. Furthermore, pregnancies in this region can show no symptoms of rupture up to 7 to 16 weeks of gestation and because of this, timely diagnosis and treatment are pivotal in this pathology due to the risk of hemorrhage and hypovolemic shock thanks to the anatomic qualities that provide its rich irrigation $[3,4,6]$. Diagnosis can be challenging, as it is based on a combination of clinical findings, laboratory tests and imaging studies $[4,6]$. Recently, assisted-reproduction techniques have increased the prevalence of ectopic pregnancies, which poses a potentially catastrophic impact on maternal death incidence due to the risk of massive haemorrhage. We present the following case-report of a interstitial ectopic pregnancy following an in vitro fertilization (IVF) pregnancy that exemplifies how an adequate prenatal control and a timely surgical approach resulted in a timely diagnosis and prevented further complications, including massive hemorrhage in this patient.

\section{Case Report}

A 32-year-old female patient with a five-year history of primary infertility, an 18 year history of lupus and a severe case of endometriosis (Stage IV), recently diagnosed laparoscopically two years ago with findings of implants in urinary bladder and rectum treated with uterine nerve ablation (LUNA). The patient also received treatment with a $3.6 \mathrm{mg}$ goserelin (zoladex) subdermic implant for 6 months. A year later, a hysterosalpingography study reported bilateral tubal 
permeability and hence, four ovulation induction cycles with programmed coitus were undertaken, without achieving pregnancy. At this time, the patient checks-in to our fertility clinic for the first time, and a gynecologic hormonal assay is performed, along with Ca-125 levels as an indirect marker for endometriosis (Table 1). She is diagnosed with primary infertility due to a tubal component and severe endometriosis. A highcomplexity cycle is initiated during the short luteal phase. During her post-cycle ultrasound (US) control, a $4.5 \times 4.1 \mathrm{~cm}$ right adnexal mass is identified with a homogenous echogenicity suggestive of an endometrioma, and an intrauterine image compatible with an endometrial polyp. The patient's Ca-125 level is $48 \mathrm{U} / \mathrm{mL}$. A laparoscopy/hysteroscopy surgery is decided, performing a polypectomy without complications, findings the presence of a right hydrosalpinx, an ipsilateral endometrioma and multiple bilateral adnexal adhesions. Hence, a bilateral tubal ligation, adhesiolysis and an endometrioma puncture were performed. Three months later, an in-vitro fecundation (IVF) is performed after pituitary suppression with leuprolide acetate. Recombinant follicle stimulant hormone (FSH) is used for ovarian hyperstimulation. Eight eggs were captured and deemed adequate for fertilization. Embryo transfer is performed, and 32 days later an ultrasonography study is performed, reporting a gestational sac of $18 \mathrm{~mm}$ and an embryo $8.2 \mathrm{~mm}$ with cardiac activity and a heterogeneous left adnexal mass of $16 \times 15 \mathrm{~mm}$. Also, an empty uterine cavity and a human chorionic gonadotropin beta fraction (hCG- $\beta$ ) of $6450 \mathrm{UI} / \mathrm{mL}$ are reported (Figure 1).

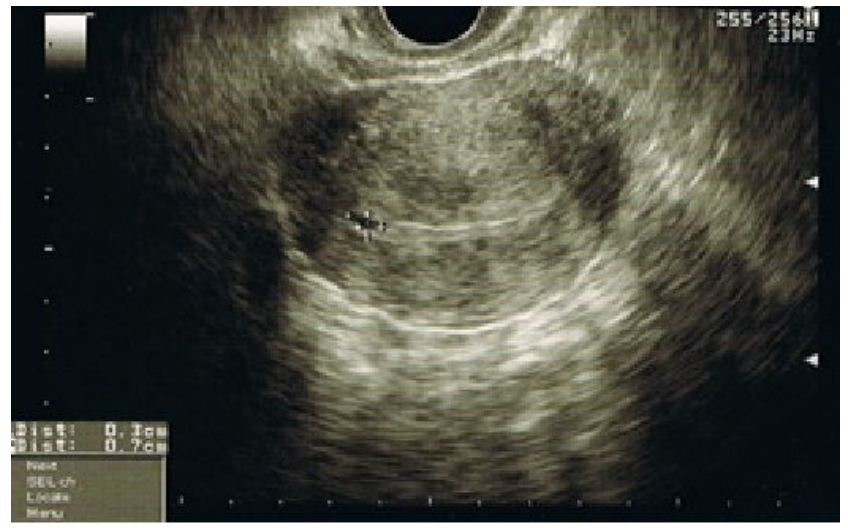

Figure 1: Tranvaginal echography empty uterine cavity.

Table 1: Gynecologic hormonal assay and Ca-125.

\begin{tabular}{|l|l|l|l|l|}
\hline FSH & LH & E2 & PRL & CA-125 \\
\hline $8 \mathrm{~m} \mathrm{UI} / \mathrm{Ml}$ & $5 \mathrm{~m} \mathrm{Ul} / \mathrm{mL}$ & $46 \mathrm{pg} / \mathrm{ml}$ & $16 \mathrm{ng} / \mathrm{ml}$ & $63 \mathrm{Ul} / \mathrm{mL}$ \\
\hline
\end{tabular}

Abbreviations: FSH: Follicle-stimulating hormone; LH: Luteinizing hormone, E2: Estradiol; PRL: Prolactin

The patient is found to be hemodynamically stable upon physical examination, without any apparent pathological process during inspection, with audible peristalsis, tender abdomen without resistance, with moderate pain at superficial palpation in the lower right abdominal quadrant without any signs peritoneal irritation. A $10 \times 5 \mathrm{~cm}$ uterus is palpated, with a $5 \times 4$ $\mathrm{cm}$ painful adnexal mass. Decision to intervene with a diagnostic/operative laparoscopy is undertaken and the procedure is carried out with a $30^{\circ}, 5 \mathrm{~mm}$ camera, and tripolar energy to perform a cornual resection and fulguration of the implantation area and tubal stump. Upon pathological analysis, an interstitial tubal pregnancy is confirmed. The patient goes through an unremarkable postoperative period and is checkedout the next day.

\section{Conclusion}

Interstitial pregnancy is also known as cornual pregnancy. However, literature sometimes differentiates between the terms by stating that a cornual pregnancy is found in the endometrium of the cornus of a bicornual/unicornual uterus, or even outside the endometrial cavity, while an interstitial pregnancy is found within the union of the uterus and the proximal portion of the Fallopian tube [6-8].

As many as $95 \%$ of ectopic pregnancies occur within the Fallopian tube, of these, $24 \%$ are interstitial, $12 \%$ isthmic, $70 \%$ ampullary, and $11.1 \%$ occur within the fimbriae $[4,7]$. The incidence of interstitial pregnancy is greater after IVF, especially in women with a history of salpingectomy due to a tubal factor $[7,8]$. Risk factors for interstitial pregnancy are similar to those of other types of ectopic pregnancy: Pelvic inflammatory disease, previous tubal surgery, previous ectopic pregnancy, and factors associated to assisted reproduction techniques, such as multiple embryo transfers, or embryos transferred in close proximity to the uterine cornua, and excessive pressure of the syringe during embryo transfer. However, bilateral tubal occlusion is the only risk factor exclusively associated to interstitial pregnancy. Moreover, in patients without bilateral tubal occlusion (BTO), peritubal or intratubal adhesions associated to endometriosis can also be a risk factor [6-9]. In the present case, the patient suffered from severe endometriosis, history of a previous tubal surgery and underwent assisted reproductive technique. As mentioned before, clinical diagnostic poses an exceptional challenge, as the distension capacity of the interstitial portion of the fallopian tube and a history of BTO can delay diagnosis and cause a critical haemorrhage in an advanced pregnancy. Moreover, 2D ultrasound can be misinterpreted in up to $20 \%$ of cases of non-broken ectopic pregnancy [7]. However, diagnostic suspicion should be made in patients with known risk factors and those who undergo assisted reproductive techniques with serial biochemical and ultrasonography controls, since early treatment allows more conservative treatments that reduce the morbidity and mortality rate. Diagnosis is accomplished by quantifying the beta fraction of the human chorionic gonadotropin, vaginal ultrasonography and sometimes even laparoscopy [9-11]. The "interstitial line" sign, part of the Trish criteria for ultrasound diagnosis was originally described by Ackerman (Table 2) $[9,11-13]$. Original treatment for interstitial ectopic pregnancy is laparotomy, hysterectomy and cornual resection, all three procedures associated to frequent complications and an increased morbidity rate [11]. Currently, there is no consensus as to which procedure is better, presently however, more conservative approaches are preferred, such 
cornuostomy and laparoscopy instead of laparotomy for fertility conservation. These procedures however, are associated in $27 \%$ to interstitial ectopic pregnancy persistence. This highlights the importance of a rigorous follow-up during the post-operative period and in future pregnancies. Furthermore, patients who are submitted to any of these treatments should undergo caesarean section because of the risk of uterine rupture $[7,8,13,14]$. As first line of treatment, the Royal College of Obstetricians and Gynaecologists (RCOG) recommends methotrexate in patients with hCG- $\beta<5000 \mathrm{UI} / \mathrm{mL}$ and ectopic pregancy smaller than 3.5 $\mathrm{cm}$ to $4 \mathrm{~cm}[9,10]$ and minimal symptoms. However, in this case, because of the hCG- $\beta$ levels and presence of cardiac activity, a surgical approach was preferred.

In conclusion, ectopic pregnancy is a complication of assisted reproductive techniques. A history of BTO does not exclude the risk of pregnancy ectopy. Interstitial pregnancy is a rare form of tubal pregnancy that poses a diagnostic challenge and is potentially life-threating due to massive haemorrhage risk, hence the need for a swift a intervention. It is thus important to encourage medical suspicion and the use of sophisticated imaging studies such as transvaginal echography to assess the precise location of an ectopic pregnancy before rupture.

Table 2: Trisch transvaginal ultrasound diagnostic criteria for of interstitial pregnancy [12].

\begin{tabular}{|c|c|}
\hline - Empty uterine cavity & \\
\hline $\begin{array}{l}\text { - Chorionic sac less than } 1 \mathrm{~cm} \\
\text { from the most lateral edge of } \\
\text { the uterine cavity. }\end{array}$ & \\
\hline $\begin{array}{l}\text { - A thick myometrial layer }(5 \mathrm{~mm}) \\
\text { which surrounds the chorionic } \\
\text { sac }\end{array}$ & \\
\hline • "Interstitial line sign" & $\begin{array}{l}\text { A thin echogenic line extends directly } \\
\text { upon the center of the interstitial } \\
\text { gestational sac in the cornual zone }\end{array}$ \\
\hline
\end{tabular}

\section{References}

1. Obajimi GO, Balogun FO, Nwose U, Obajimi OM (2013) Right ampullary ectopic pregnancy following bilateral tubal ligation using spring clips. Ann Ibd Pg Med 11: 34-36.

2. Álvarez-Goris $M$ del $P$, Mendoza-Moreno $J M$, Sánchez-Zamora $R$, Torres-Rivera RA, Galicia-Pedraza AK (2015) Ovarian ectopic pregnancy with little common clinical presentation. A case report and bibliographical review. Ginecol Obstet Mex 83: 545-550.

3. Moawad NS, Mahajan ST, Moniz MH, Taylor SE, Hurd WW (2010) Current diagnosis and treatment of interstitial pregnancy. Am J Obstet Gynecol 202: 15-29.

4. Garavaglia E, Quaranta L, Redaelli A, Colombo G, Pasi F, et al. (2012) Interstitial pregnancy after in vitro fertilization and embryo transfer following bilateral salpingectomy: report of two cases and literature review. Int J Fertil Steril 6: 131-134.

5. Ahn JW, Lee SJ, Lee SH, Kang SP, Won HS (2013) Ultrasound-guided transcervical forceps extraction of unruptured interstitial pregnancy. BJOG 120: 1285-1288.

6. Grindler NM, Ng J, Tocce K, Alvero R (2016) Considerations for management of interstitial ectopic pregnancies: two case reports. J Med Case Rep 10: 106.

7. Wang J, Huang D, Lin X, Saravelos SH, Chen J, et al. (2016) Incidence of Interstitial Pregnancy After In Vitro Fertilization/ Embryo Transfer and the Outcome of a Consecutive Series of 38 Cases Managed by Laparoscopic Cornuostomy or Cornual Repair. J Minim Invasive Gynecol 9: 134-135.

8. Warda H, Mamik M, Ashraf M, Abuzeid M (2014) Interstitial Ectopic Pregnancy: Conservative Surgical Management. JSLS 18 197-203.

9. Sharma N, Upasana R (2013) An ectopic pregnancy in the tubal interstitium: Beware! J Clin Diagn Res 7: 160-162.

10. Cucinella G, Calagna G, Rotolo S, Granese R, Saitta S, et al. (2014) A Interstitial Pregnancy: A 'Road Map' of Surgical Treatment Based on a Systematic Review of the Literature. Gynecol Obstet Invest 78: 141-149.

11. Singh N, Tripathi R, Mala YM, Batra A (2015) Diagnostic dilemma in cornual pregnancy-3D ultrasonography may Aid!! J Clin Diagn Res 9: 12-13.

12. Tritsch IET, Monteagudo A, Lerner JP (1997) A "potentially safer" route for the puncture and the injection of cornual ectopic pregnancies. Ultrasound Obstet Gynaecol 7: 353-355.

13. Surbone A, Cottier O, Vial Y, Francini K, Hohlfeld P, et al. (2013) Interstitial pregnancies' diagnosis and management: an eleven cases series. Swiss Med Wkly 143: 13736.

14. Ackerman TE, Levi CS, Dashefsky SM, Holt SC, Lindsay DJ (1993) An interstitial line: sonographic finding in an interstitial (cornual) ectopic pregnancy. Radiology 189: 83-87. 